\title{
Further development of hybrid functional electrical stimulation orthoses
}

\author{
L Yang ${ }^{1}$, MH Granat ${ }^{1}$, JP Paul ${ }^{1}$, DN Condie ${ }^{2}$ and DI Rowley ${ }^{3}$ \\ ${ }^{1}$ Bioengineering Unit, University of Strathclyde, Glasgow G4 ONW, UK; ${ }^{2}$ Dundee Limb Fitting Centre, 133 Queen \\ Street, Broughty Ferry, Dundee, UK; ${ }^{3}$ Department of Orthopaedic \& Trauma Surgery, Dundee University, D.R.I., \\ Dundee, UK
}

\begin{abstract}
It is recognised that, in paraplegic walking with reciprocating gait orthoses, inadequate hip flexion angles may contribute to the low walking speed and high energy cost. In this study a new orthotic hip joint was developed which had a 2:1 flexion extension coupling ratio. Experiments on paraplegic subjects were conducted to evaluate this orthotic hip joint. It was found that the new hip joint was associated with a reduced energy cost and increased step length. A simple application of FES assisted hip flexion further increased walking speed and step length and reduced energy cost and crutch force impulse.
\end{abstract}

Keywords: functional electrical stimulation; reciprocating gait orthosis; paraplegia; orthotic hip joint

\section{Introduction}

Modern mechanical orthoses such as the ORLAU ParaWalker $^{1}$ and the Louisiana State University Reciprocating Gait Orthosis $(\mathrm{RGO})^{2}$ have been widely used to provide limited walking for paraplegic persons. These systems combine trunk and leg braces to prevent collapse of the lower limbs and the wearer achieves forward progression using elbow crutches or a walking frame to raise the trunk and to allow the swinging leg to progress forward. The energy cost of reciprocal gait for paraplegic individuals is up to six times the energy cost for using a wheelchair, and the speed of walking is about a quarter the speed of wheelchair ambulation, so that the users gain only limited functional benefits. ${ }^{3.4}$

In order to enhance the efficiency of paraplegic walking, functional electrical stimulation (FES) has been used in association with mechanical orthoses. Solomonow $^{5}$ described a hybrid FES orthosis system consisting of an RGO and bilateral stimulation of the rectus femoris and the hamstring muscles. $\mathrm{He}$ demonstrated that compared with pure RGO and pure FES walking systems, the hybrid FES system had the lowest energy cost at the paraplegic person's preferred walking speed of $0.2 \mathrm{~m} / \mathrm{s}$. Nene and Patrick ${ }^{6}$ stimulated the gluteal muscles in conjunction with the use of the ParaWalker and found a reduction in energy cost of 6-9\%. Although these studies provided encouraging results, the high energy cost of paraplegic walking remains an important factor which severely limits the use of the systems.

Most hybrid FES systems adopt a simple 'stiff-leg' reciprocal walking mode. In this mode hip flexion is the minimal prerequisite and the step length is directly related to the angle between the flexed and extended

Correspondence: L Yang, Clinical Sciences Centre, Northern General Hospital, Sheffield S5 7AU thighs at heel strike. It was observed that paraplegic walking with an RGO or ParaWalker has inadequate hip flexion leading to very short step length. ${ }^{7}$ It is difficult to achieve pure hip flexion by stimulating hip flexors using surface electrodes, while the stimulation of the lower limb withdrawal reflex has shortcomings of variable response and habituation. ${ }^{8}$ To resolve the problem, a new coupling mechanism for the RGO hip joints was developed based on normal walking data. The new hip joint used a 2:1 hip flexion extension coupling ratio (FECR) as defined below. This was evaluated on three able bodied subjects showing that the 2:1 hip ratio was associated with $20 \%$ lower Physiological Cost Index ${ }^{9}$ (PCI), 5\% higher speed and $6 \%$ longer stride length when compared with the $1: 1$ hip ratio. ${ }^{10}$

This article firstly gives a description of the new hip coupling mechanism followed by a report on the performance of the hip joint on three paraplegic volunteers, both with and without FES. The possibility of employing the FES to control a free knee joint is also investigated.

\section{Methods}

The new hip joint and the assessment orthosis

Based on the consideration that in 'stiff leg' walking the step length is proportional to the angle between the extended hip and the contralateral flexed hip at heel strike, the hip flexion extension coupling ratio (FECR) was defined as the ratio at heel strike of one hip flexion angle to the extension angle of the other. In normal walking, this ratio is about 2:1 as shown in Figure 1(a) (note the arrows). In the RGO the ratio is $1: 1$ and this may limit the step length. The principal aim of the new 
hip coupling mechanism developed in this study was to increase the FECR. Figure 2 is a schematic presentation of the mechanism. As the arrows in the figure show, when the left leg extends, the driving plate $4_{L}$, which is locked to the thigh member $2_{\mathrm{L}}$, also rotates in the same direction, driving the pin $6_{\mathrm{L}}$ up the guiding slot $3_{\mathrm{L}}$. The connecting push-pull cable transfers the movement by pushing the right driving pin $6_{R}$ down the guiding slot $3_{R}$, causing the plate $4_{R}$ to rotate in the opposite direction to the plate $4_{\mathrm{L}}$, as does the right leg $2_{R}$, ie, the right leg flexes. Since the driving pin $6_{R}$ is closer to the hip joint centre than pin $6_{\mathrm{L}}$, a greater flexion angle is produced on the right side. The coupling mechanism could be disengaged by releasing the lock $7_{L}$ and $7_{R}$ between the thigh member and the driving plate, allowing both legs to flex or extend for sitting down and standing up. The exact FECR is dependent on the orientation angle of the guiding slot which can be adjusted by rotating the circular plates. Figure 1(b) illustrates the theoretical hip angle-angle diagram of this new coupling mechanism.

An assessment brace was developed to impose joint motion constraints. It had uniaxial knee joints with drop locks, uniaxial modular ankle joints allowing free plantar flexion, free dorsi flexion, free or fixed joints, and the new hip joints which could simulate the hip joints of the ParaWalker (free hip) and the RGO with a pre-set FECR.

\section{Patient study}

Three paraplegic volunteers attending the Queen Elizabeth National Spinal Injuries Unit participated in the study, and their particulars are listed in Table 1. The study was approved by the local hospital Ethics Committee. All protocols were explained to, and an informed consent was obtained from, each subject. Muscle conditioning using FES was prescribed when necessary.

\section{A Angle-Angle Diagram} (normal walking)

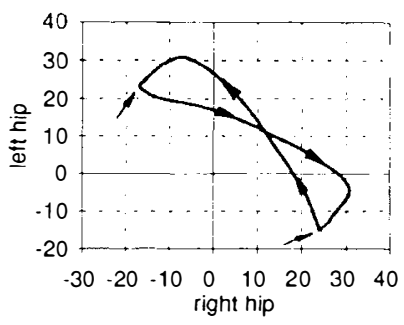

B Angle-Angle Diagram (theoretical)

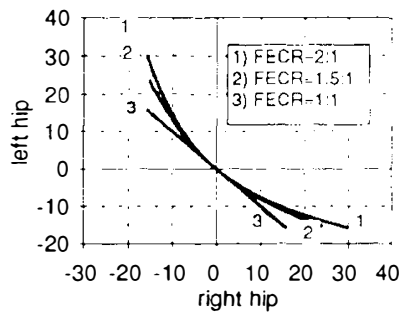

Figure 1 (a) Relationship between flexion and extension angles of left and right hips in normal walking. The points corresponding to heel strike are marked by arrows. This data was obtained using VICON-VX system (Oxford Metrics, England) from a normal subject walking at his preferred speed. (b) Theoretical relationship between flexion and extension angles of left and right hips of the new coupling mechanism
Two series of experiments were conducted. In the first series, the effects of different hip FECR (1:1 or $2: 1)$ on the paraplegic walking were investigated. In these tests, no FES was used. The subjects were unaware of which hip FECR was being used. In the second series of experiments, the performance of paraplegic walking with and without FES assisted hip flexion was studied. In these tests, the hip joints were set to 2:1 FECR due to its superior performance over 1:1 (see Results). To assist hip flexion, the common peroneal nerve was stimulated which evoked the flexion withdrawal reflex. For one subject, the peroneal stimulation produced an inappropriate response and therefore rectus femoris was stimulated instead. The stimulation parameters consisted of $25 \mathrm{~Hz}$ monophasic, square pulses with a pulse width of $300 \mu \mathrm{s}$. The onset and duration of stimulation was controlled by a switch mounted on the handle of each elbow crutch. Each series of tests consisted of two experimental sessions and each session consisted of at least four walking trials for each orthotic configuration or each FES control strategy.

A walking trial consisted of walking along a straight

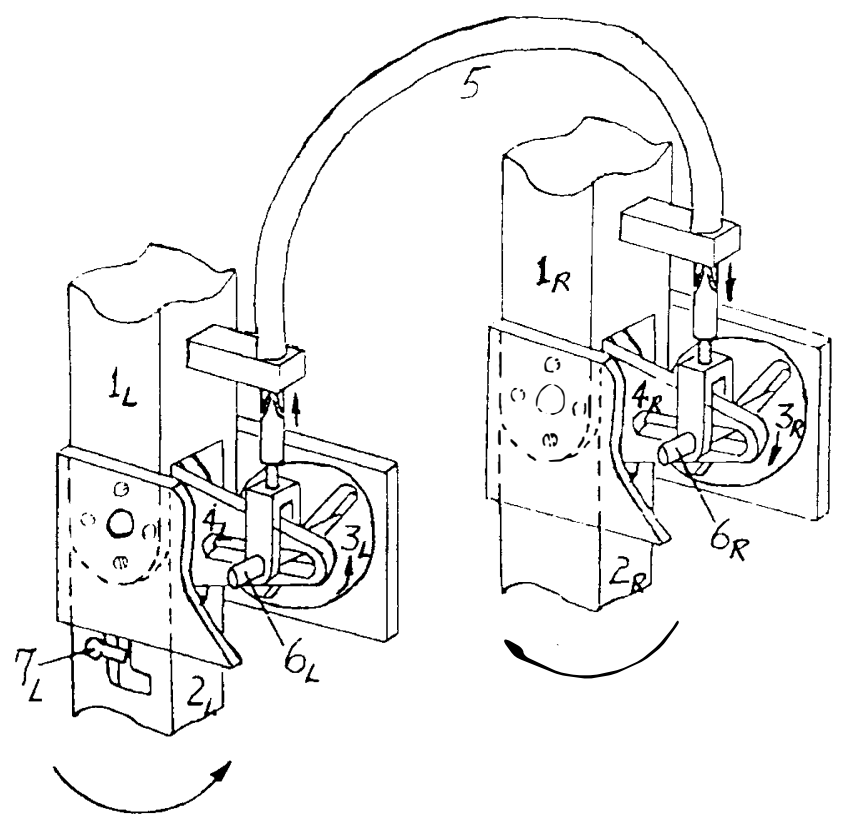

Figure 2 A schematic diagram of the orthotic hip coupling mechanism. $1_{L}$ and $1_{R}$ represent parts of the pelvic component of the orthosis. Other components are described in the text

Table 1 Test subject profile

\begin{tabular}{lllll}
\hline $\begin{array}{l}\text { Subject } \\
\text { code }\end{array}$ & Age & $\begin{array}{l}\text { Level of } \\
\text { lesion }\end{array}$ & $\begin{array}{l}\text { Date of } \\
\text { injury }\end{array}$ & $\begin{array}{l}\text { Walking } \\
\text { aids }\end{array}$ \\
\cline { 3 - 3 } A & 42 & T4 complete & 1985 & elbow crutch \\
B & 33 & C6 incomplete & 1981 & elbow crutch \\
C & 28 & T8 complete & 1993 & parallel bars \\
\hline
\end{tabular}


path of length of $6.5 \mathrm{~m}$ for subjects $\mathrm{A}$ and $\mathrm{B}$ who walked with crutches, or $2.5 \mathrm{~m}$ for subject $\mathrm{C}$ who walked in parallel bars. Rotary potentiometers were used to measure the hip angles. A pair of elbow crutches were strain gauged to measure crutch axial forces, and crutch impulse was calculated by integrating the crutch force by time during a gait cycle. To calculate the PCI, the Sport Tester PE3000 (Polar Electro, Finland) was used to record the heart rate. This technique was employed by other investigators on normals and paraplegics. ${ }^{11-13}$ A stop watch was used to measure the time required to cover the measured distance. The number of steps to cover the distance was counted and the average step length was then calculated

\section{Results}

In the first series of experiments where the effect of hip FECR was studied, all subjects felt that the leg was easier to swing through with 2:1 FECR. Referring to Table 2, the 2:1 FECR was associated with, on average, $15 \%$ lower PCI and 4\% longer stride lengths when compared with the 1:1 FECR, although this was not always statistically significant $(P<0.05)$. The effects of hip configuration on the walking speed were variable between different days and subjects. Increases in walking speed did not always coincide with the increases in stride length, suggesting that in these cases the cadence was increased. From the crutch force data, it appeared that 2:1 FECR was related to higher crutch impulses compared with the 1:1 FECR.

In the second series of tests, as showed in Table 3, the FES assisted hip flexion, compared with no FES, was associated with significantly $(P<0.05)$ reduced PCI and crutch force impulse and with increased walking speed and stride length. On average, the subjects tested with FES assisted hip flexion walked $13 \%$ faster, with $42 \%$ lower PCI, $17 \%$ lower crutch impulses and $5 \%$ longer stride length.

\section{Discussion}

The hypothesis tested in this programme was that the new orthotic hip joints providing 2:1 FECR would increase step length and walking speed leading to reduction in energy cost. The result of the patient study confirmed that longer step lengths and lower energy cost were associated with the 2:1 hip FECR. However the walking speeds did not increase consistently with the 2:1 FECR, suggesting that the reduction in PCI was due to the decreased heart rate. It was possible that the efficiency of the energy transfer from the trunk to the lower limbs via the hip joints was increased with the 2:1 FECR. Bernardi et al ${ }^{14}$ recently showed that the amount of transfer between kinetic and potential energy in paraplegic RGO walking was greatly reduced compared with normal walking and stipulated that energy transfer from the trunk movement to the lower limb via the RGO hip joint was inefficient. It should be pointed out that although there was a $15 \%$ reduction in PCI with 2:1 FECR the energy cost was still high compared with normal walking.

It appeared from the results that the 2:1 FECR was related to higher crutch impulses compared with the 1:1 FECR. This finding should be interpreted in the context that the corresponding walking speeds were reduced. An increased step length would result in the centre of mass of the torso being lower and further back at heel strike. With a decreased walking speed there would be less energy transfer between kinetic and potential energy of the torso. As a consequence of these two factors, more effort per step from the upper limb muscles would be needed to move the torso upward and forward on to the stance leg.

The present study showed that FES assisted hip flexion markedly improved paraplegic ambulation efficiency compared with the use of the purely mechanical orthosis. This result is in agreement with those of Solomonow ${ }^{5}$ and Nene and Patrick, ${ }^{6}$ and supports the belief that the use of FES applied to the

Table 3 Effects of hip flexor stimulation on paraplegic walking

\begin{tabular}{|c|c|c|c|c|c|c|c|c|}
\hline \multirow[b]{3}{*}{ FES applied } & \multicolumn{4}{|c|}{ Subject $A$} & \multicolumn{4}{|c|}{ Subject $B$} \\
\hline & \multicolumn{2}{|c|}{ Session 1} & \multicolumn{2}{|c|}{ Session 2} & \multicolumn{4}{|c|}{ Session 1 Session 2} \\
\hline & No & Yes & No & Yes & No & Yes & No & Yes \\
\hline PCI (beat $/ \mathrm{m})$ & 5.2 & 2.8 & 5.3 & 1.1 & 4.2 & 2.8 & 3.7 & 3.3 \\
\hline Speed $(\mathrm{m} / \mathrm{s})$ & $\overline{0.18}$ & $\overline{0.20}$ & $\overline{0.19}$ & $\overline{0.22}$ & $\overline{0.31}$ & $\overline{0.34}$ & 0.31 & 0.36 \\
\hline Stride length $(\mathrm{m})$ & 0.74 & 0.78 & 0.76 & 0.82 & 0.98 & 0.96 & 0.94 & 1.12 \\
\hline Crutch impulse (Ns) & 617 & 445 & $\overline{454}$ & $\overline{434}$ & 420 & 327 & 431 & 367 \\
\hline
\end{tabular}

Table 2 Effects of different hip flexion extension coupling ratios on paraplegic walking

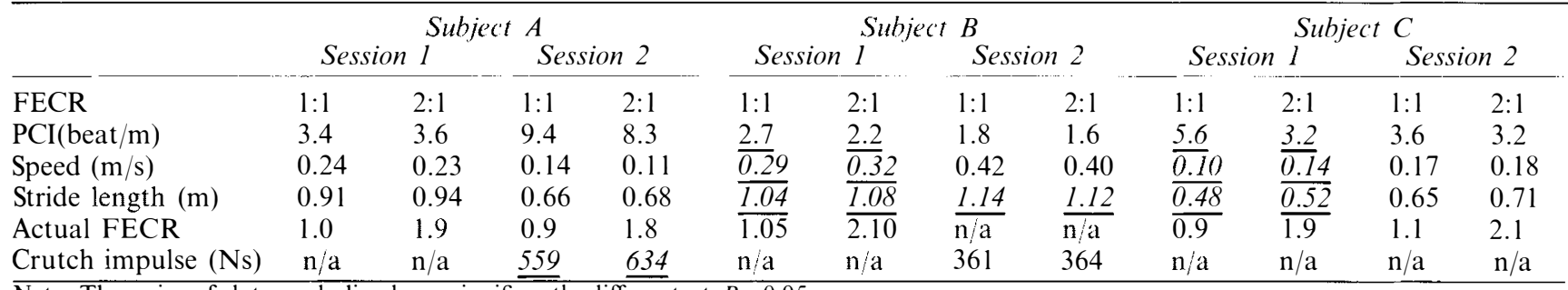

Note: The pairs of data underlined are significantly different at $P<0.05$ 
lower limb muscles would reduce the upper limb muscle forces. Since the lower limb muscles are better adapted to such loading than the upper limb muscles, the overall energy required for locomotion would be decreased.

In normal walking knee flexion during swing phase reduces the elevation of the body's centre of mass. With a fixed knee the centre of mass has to be raised by the stance leg to ensure the ground clearance of the swing leg, requiring extra energy to do this. The study on able bodied subjects confirmed that free knee ambulation was a desirable means of reducing the energy cost of locomotion. ${ }^{10}$ Limited patient trials were attempted by the authors on subject B to implement an FES controlled free knee joint configuration. This turned out to be quite difficult. To ensure a stable knee, quadriceps muscles were stimulated throughout the stance phase, leading to more rapid fatigue. The anatomical knee joint is polyaxial, while the orthotic knee joint is uniaxial, so that the two knee joints did not coincide with each other, producing a force resisting knee flexion and extension. It is desirable to have an orthotic knee joint which can be locked in extension during stance and free during swing phase. Bataweel and Edwards ${ }^{15}$ recently reported a hybrid FES orthosis system with such a knee joint with which an $18 \%$ reduction in PCI and $50 \%$ reduction in rollator vertical force were achieved.

\section{Conclusion}

The new orthotic hip joint with 2:1 hip flexion to extension coupling ratio was associated with a moderate reduction in energy cost and with a slight increase in step length. An application of FES assisted hip flexion markedly reduced energy cost, moderately reduced crutch impulses and increased walking speed.

\section{Acknowledgements}

This study was funded by Action Research $(\mathrm{A} / \mathrm{P} / 0331)$ and this is gratefully acknowledged. We also sincerely thank Mr Edmond, Mr Hasler and the subjects at the Queen Elizabeth National Spinal Injuries Unit for their generous help throughout this study.

\section{References}

1 Butler PB, Major RE. The ParaWalker: A rational approach to the provision of reciprocal ambulation for paraplegic patients. Physiotherapy 1987; 73: 393-397.

2 Douglas R, Larson PF, D'Ambrosia R, McCall RE. The LSU reciprocal gait orthosis. Orthopedics 1983; 6: $834-838$.

3 Nene AV, Patrick JH. Energy cost of paraplegic locomotion with ORLAU ParaWalker. Paraplegia 1989; 26: 5-18.

4 Bowker P, Messenger N, Ogilvie C, Rowley DI. Energetics of paraplegic walking. J Biomed Eng 1992; 14: $344-350$.

5 Solomonow M. Biomechanics and physiology of a practical functional neuro-muscular stimulation powered walking orthosis for paraplegics. In Neural Prostheses (ed). RB Stein, PH Peckham, DP Popovic, Oxford University Press: Oxford, 1992; pp 202-232.

6 Nene AV, Patrick JH. Energy cost of paraplegic locomotion using the ParaWalker-electrical stimulation 'hybrid' orthosis. Arch Phys Rehabil 1990; 71: 116-120.

7 Jefferson RJ, Whittle MW. Performance of three walking orthoses for the paralysed: a case study using gait analysis. Prosth Orth Int 1990; 14: $103-110$.

8 Granat $\mathrm{MH}$ et al. Improving limb flexion in FES gait using the flexion withdrawal response for the spinal cord injured person. $J$ Biomed Eng 1993; 15: 51 - 56.

9 MacGregor J. The objective measurement of physical performance with long-term ambulatory surveillance equipment. Proc 3rd Int Symp Ambulatory monitoring 1979; Academic Press, pp $29-39$.

10 Yang L et al. Effects of joint motion constraints on the gait of normal subjects and their implications on the further development of hybrid FES orthosis for paraplegic persons. J Biochem 1996; 29: $217-226$

11 Leger L, Thiverge M. Heart rate monitors: validity, stability and functionality. Physician \& Sports Med 1988; 16: $143-151$.

12 Bar-On Z, Nene AV. Relationship between heart rate and oxygen uptake in thoracic level paraplegics. Paraplegia 1990; 28: $87-95$

13 Tsanakas JN, Bannister OM, Boon AW, Milner RDG. The 'Sport Tester': a device for monitoring the free running test. Arch Dis Childhood 1986; 61: 912 -914.

14 Bernardi $\mathrm{M}$ et al. The efficiency of walking of paraplegic patients using a reciprocating gait orthosis. Paraplegia 1995; 33: $409-$ 415.

15 Bataweel AO, Edwards J. An orthotic/FES system for paraplegic walking with a knee bending swing phase. Proc BES Symposium on Electrical Stimulation - Clinical Systems; Glasgow, 1995, pp $7-8$. 